\title{
Ultrasound-guided internal jugular vein cannulation: a novel technique for confirming that the guidewire is directed towards the right atrium
}

\author{
Praveen Maheshwari, MD • Parul Maheshwari, MD
}

Received: 22 August 2015/Revised: 24 September 2015/Accepted: 14 October 2015/Published online: 27 October 2015

(C) Canadian Anesthesiologists' Society 2015

\section{To the Editor,}

We read the article by Aoyama et al. ${ }^{1}$ regarding guidewire confirmation during ultrasound-guided internal jugular vein (IJV) cannulation and offer an additional suggestion for the use of ultrasound (US) after identifying the IJV and placing a guidewire. Although several techniques have been described ${ }^{1,2}$ to address confirmation of the location of the guidewire in the IJV, another issue may be encountered during IJV cannulation. After confirming the guidewire's location in the IJV, difficulty may be encountered in directing the guidewire towards the right atrium (RA) and then in advancing the central venous catheter (CVC) tip towards the junction of the superior vena cava (SVC) and RA. Indeed, there have been numerous reports of misplacement of CVCs. ${ }^{3,4} \mathrm{We}$ suggest a simple maneuver to supplement correct IJV cannulation and subsequent $\mathrm{CVC}$ advancement towards the RA.

Our technique entails placing a linear US probe over the IJV perpendicular to the guidewire (Figure A). With this approach, a short-axis view of the IJV is obtained, allowing confirmation of the guidewire's location within the IJV lumen (Figure B). The US probe can then be directed caudally until it reaches the superior edge of the clavicle, at which point it is gradually tilted posteroinferiorly towards the thoracic cavity (Figure C). With the US probe in this position, a long-axis (albeit somewhat foreshortened) view of the SVC can be visualized and the location of the guidewire within the lumen of the SVC can be confirmed (Figure D). The depth of the US sector and the angulation of the US probe may need to be adjusted in order to visualize deeper structures such as the more distal SVC. This novel maneuver allows for dynamic visualization of the guidewire directed through the SVC towards (and even into) the RA and may help decrease the incidence of incorrectly positioned CVCs.

In a similar fashion, Arellano et al. ${ }^{5}$ reported the utility of transthoracic echocardiography (TTE) for confirming the location of the guidewire in the RA before dilation and cannulation of the IJV. Nevertheless, the disadvantage of using TTE for guidewire confirmation is the need for sterile preparation of another ultrasound probe, expertise to perform TTE, and additional time to complete the procedure.

Our suggested maneuver would be advantageous in providing quick and dynamic confirmation that the guidewire is directed towards or even into the RA. 

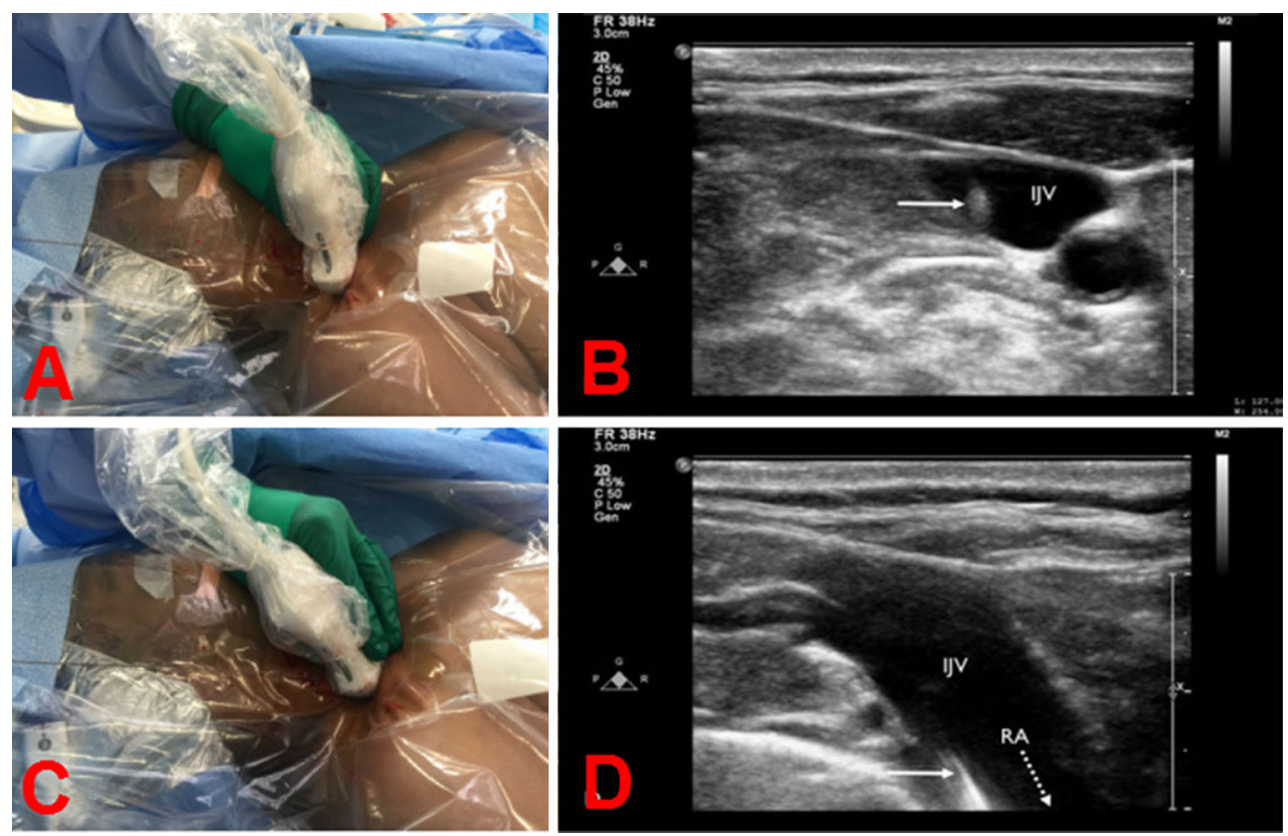

Figure A linear ultrasound (US) probe is placed over the right internal jugular vein (IJV) perpendicular to the guidewire (Figure A) producing a short-axis image (Figure B) of the IJV and the guidewire (solid white arrow). By tilting the ultrasound probe in a

posteroinferior direction towards the thoracic cavity (Figure C), a foreshortened view (Figure D) of the junction of the IVC with the superior vena cava can be obtained, confirming that the guidewire is positioned in the direction of the right atrium (dotted white arrow)

Conflicts of interest None declared.

\section{References}

1. Aoyama K, Takenaka I, Iwagaki T, Sano H. A simple maneuver for confirmation of the guidewire during ultrasound-guided internal jugular vein cannulation. Can J Anesth 2015; 62: 839-40.

2. Maheshwari $P$, Maheshwari $P$. Ultrasound-guided internal jugular vein cannulation: techniques to confirm guidewire location. Can J Anesth 2015; DOI:10.1007/s12630-015-0466-0.

3. Yang SH, Jung SM, Park SJ. Misinsertion of central venous catheter into the suspected vertebral vein: a case report. Korean J Anesthesiol 2014; 67: 342-5.

4. Calvino J, Bravo J, Martinez L, Millan B, Pulpeiro JR. Recognizing misplacement of a dialysis catheter in the azygos vein. Hemodial Int 2013; 17: 455-7.

5. Arellano R, Nurmohamed A, Rumman A, Day AG, et al. The utility of transthoracic echocardiography to confirm central line placement: an observational study. Can J Anesth 2014; 61: 340-6. 